\title{
Weak-coupling approach to the semi-infinite Hubbard model: Non-locality of the self-energy
}

\author{
M. Potthoff and W. Nolting \\ Lehrstuhl Festkörpertheorie, Institut für Physik, Humboldt-Universität zu Berlin, Germany
}

\begin{abstract}
The Hubbard model on a semi-infinite three-dimensional lattice is considered to investigate electron-correlation effects at single-crystal surfaces. The standard second-order perturbation theory in the interaction $U$ is used to calculate the electronic self-energy and the quasi-particle density of states (QDOS) in the bulk as well as in the vicinity of the surface. Within a realspace representation we fully account for the non-locality of the self-energy and examine the quality of the local approximation. Numerical results are presented and discussed for the three different low-index surfaces of the simple-cubic lattice. Compared with the bulk significant differences can be found for the top-layer local self-energy, the imaginary part of which is energetically narrowed and has a reduced total weight. The non-local parts of the self-energy $\Sigma_{i j}(E)$ decrease with increasing distance between the sites $i$ and $j$. At the surface and for the three-dimensional bulk their decrease is faster than for a two-dimensional lattice. For all surfaces considered the effects of the non-local parts of the self-energy on the QDOS are found to be qualitatively the same as for the bulk: The weight of the quasiparticle resonance at the Fermi energy is lowered while the high-energy charge-excitation peaks become more pronounced. The main structures in the layer-dependent spectra are already recovered within the local approximation; taking into account the nearest-neighbor non-local parts turns out to be an excellent approximation. Due to the reduced coordination number for sites at the very surface, the top-layer QDOS is narrowed. Contrary to the the free $(U=0)$ system, quasi-particle damping results in a comparatively weak layer dependence of the QDOS generally. Pronounced surface effects that are related to surface Friedel oscillations show up as a fine structure within the resonance around the Fermi level.
\end{abstract}

PACS: 71.27.+a, 73.20.At, 75.10.Lp

\section{INTRODUCTION}

The Hubbard model [1, 2, 3] is one of the most extensively studied models for interacting fermions on a lattice. While originally it was set up to study the conditions for metal-insulator (Mott) transitions and spontaneous magnetism, it nowadays quite generally contributes to the basic understanding of different types of prominent correlation effects.

The mutual influence of electron correlations and the geometry of the underlying lattice is one of the central aspects of recent research. The simplest example for the decisive role of the geometry is given by the lattice dimension $D$. In the extreme cases $D=1$ [4, 同, 河 and $D=\infty$ 迆, 8, 9] the Hubbard model exhibits completely different physical properties. Also for a given dimension, say $D=3$, the lattice structure is important. The exact results by Nagaoka [10] as well as approximate treatments 111, 12, 13 represent illustrating examples for the interplay between magnetic order and the lattice type.

The limit $D=\infty$ is of special interest since here the effects of the lattice geometry are suppressed as much as possible while the model itself remains non-trivial. The on-site Green function $G_{i i \sigma}(E)$ depends on the geometrical structure merely via the free Bloch-density of states (BDOS), and the self-energy $\Sigma_{\sigma}(E)$ becomes k-independent or site-diagonal 14. However, even for $D=\infty$ the dependence on the lattice type, i. e. on the BDOS is important as has been proved, for example, by the recent investigations on ferromagnetism for the hypercubic and an fcc-type lattice 15, 16].

It has been argued [8] that the essential physical properties of the $D=\infty$ Hubbard model are comparable to those for finite dimensions. A solution of the $D=\infty$ Hubbard model would thus provide for a proper meanfield theory. Actually this argument can be considered as a justification of the local approximation for the selfenergy, i. e. the neglect of its $\mathbf{k}$-dependence or its nonlocal parts in the case of finite dimension. Since the contribution of non-local parts of the self-energy to relevant physical quantities scales with $1 / D[\mathbb{8}]$, the local approximation becomes less accurate in the opposite limit $D \mapsto 1$. Starting from the local approximation and successively including nearest-neighbor, next-nearest neighbor and further correction terms, corresponds to an expansion in $1 / D$. An instructive demonstration of this fact has been worked out by Schweitzer and Czycholl 117] for $D=1-3$. The expansion converges even for $D=1$; for $D=2$ all corrections beyond the 5 -th neighbor shell are found to be negligibly small, and for $D=3$ 
the local approximation is quite appropriate.

The interplay between geometric and electronic structure becomes more important and interesting when the translational symmetry of the lattice is incomplete. This situation is realized e. g. in the case of a film of finite thickness composed of two-dimensional layers or at the surface of a three-dimensional lattice. Our study aims at the latter case, the semi-infinite Hubbard model. The semi-infinite Hubbard model may be interesting for different reasons. First of all, it comes closer to the experimental situation. Because of the finite inelastic mean free path of low-energy electrons in the solid [18], surface effects are often non-negligible or even dominating in electron spectroscopy. Furthermore, surface effects may be interesting of their own. Particularly, the semiinfinite Hubbard model can contribute to the understanding of phenomena related to surface magnetism. The combined influence of electron-correlation as well as of surface effects is important for the study of magnetic surface states, of magnetic moments at the surface and a possibly enhanced surface Curie temperature etc. To our knowledge little effort has been spent on these topics up to now; the investigation of itinerant electron models with reduced translational symmetry (thin films, surfaces) is still at the very beginning (see Refs. $19,20,21,22,23])$. On the other hand, there has been extensive work on the semi-infinite Ising and Heisenberg model [24]. These localized spin models, however, have to be considered as effective models where the magnetic order is more or less pretexted rather than derived consistently from the electronic structure.

Despite its conceptual simplicity, an exact solution of the Hubbard model or an approximation scheme that is reliable in the entire parameter space is not available for $D=3$ up to now. The presence of the surface and the corresponding breakdown of translational symmetry in the surface normal direction still complicates the problem, and thus approximations must be tolerated. One of the main purposes of the present paper is to contribute to a clarification of the status of the local approximation for the self-energy in the context of the semi-infinite Hubbard model. This promises to be an interesting question since a priori it is not obvious whether a solid surface in this respect should be regarded as being close to $D=2$ or close to $D=3$. However, while the local approximation may be sufficient in the case $D=3$, it is less accurate in $D=2$ 17, 25, 26. It can be expected that the degree of nonlocality of the self-energy will be tightly connected with the reduction of the coordination number of sites at the very surface. One should try to substantiate a reasoning based on coordination numbers by means of a more quantitative analysis. This includes the investigation of different (low-index) surfaces of the same lattice. While non-local parts $\Sigma_{i j \sigma}(E)$ for $i \neq j$ are expected to decrease for distances $\left\|\mathbf{R}_{i}-\mathbf{R}_{j}\right\| \mapsto \infty$, the number of atoms within a shell of increasing radius $R$ around the site $i$ may increase. This raises the question of the effect of non-local contributions on the quasi-particle density of states and the changes near the surface.

In the present paper we try to tackle the mentioned problems within the weak-coupling approach to the semi-infinite Hubbard model, i. e. within second-order perturbation theory (SOPT) in the interaction $U$. For the translationally symmetric Hubbard model this is a standard approximation scheme 117, 27, 28, 29, 30, 31. 32, 33, 34, 35. Its numerical evaluation in $\mathbf{k}$-space includes non-trivial multiple integrals. An efficient realspace algorithm for the calculation of the self-energy $\Sigma_{i j \sigma}(E)$ was introduced in Refs. 17, 36 and is easily seen to be applicable also in the case of reduced translational symmetry. The SOPT will yield exact results for infinitesimal $U=0^{+}$and will give first hints at least for finite $U$. An extrapolation of the results to strong interaction $U$ (compared with the Bloch-band width $W$ ) must be questioned, of course. On the other hand, the comparison of the SOPT results for moderate interaction $U$ [33] with the results of a strong-coupling moment method 11] is encouraging since qualitatively both approaches agree well. In Ref. [33] Bulk and Jelitto employed the SOPT around the Hartree-Fock (HF) solution. This approach turns out to be superior compared with the plain SOPT in which free instead of HartreeFock propagators are used in the calculation of the proper irreducible self-energy diagram. Neither plain SOPT nor SOPT-HF, however, is a conserving approximation in the sense of Baym and Kadanoff [37]. Contrary, the self-consistent SOPT (using the fully renormalized propagators) does represent a $\Phi$-derivable theory [32, 38].

Most of the results presented here have been calculated for $U=0^{+}$. In this case the different versions of the weak-coupling theory become identical. Considering an infinitesimal interaction, however, does not allow to study the manifestation of correlation effects in the quasi-particle density of states. Therefore, we will also consider finite interaction $U$, but restrict ourselves to the symmetric situation of a paramagnet at half-filling $\left(n \equiv 2\left\langle n_{i \sigma}\right\rangle=1\right)$ in this case. The symmetric case is somehow exceptional; the following well-known arguments substantiate that SOPT-HF may yield qualitatively correct results even for moderate interaction $U \sim W$ at $n=1$. Firstly, SOPT-HF exactly reproduces the atomic limit $(W=0)$ at half-filling. Secondly, the energetic positions as well as the spectral weights of both high-energy charge-excitation peaks that show up in the spectrum for $U \gg W$ can be calculated exactly within a strong-coupling perturbation approach (expansion in $W / U)[39,40]$. For the case of half-filling SOPT-HF can easily be shown to reproduce these exact results. We thus believe that the method reasonably interpolates between the weak- $(U \ll W)$ and the strong-coupling $(U \gg W$ and $W=0)$ regimes. On the contrary, the self-consistent SOPT fails with respect to all points mentioned. In our opinion this cannot be outweighted by its advantages, namely to represent a conserving theory 
and thus to respect the Luttinger sum rule 443. Since the SOPT-HF scheme preserves particle-hole symmetry 44, the sum rule is fulfilled anyway in the case of halffilling. The last argument regards the high-energy behavior of the SOPT-HF self-energy. At half-filling the first two coefficients (for the local part three coefficients) within an $1 / E$ expansion turn out to be exact (see Ref. 41, 42 ). This also implies that the first three moments of the quasi-particle density of states can be reproduced. Another reason for using SOPT-HF is a pragmatic one: For the general (non-symmetric) case, self-consistency with respect to the occupation number $\left\langle n_{i \sigma}\right\rangle$ and the chemical potential $\mu$ is required. At half-filling this is achieved trivially, we have $\mu=U / 2$ (this also holds for the semi-infinite lattice). Since our main interest is focussed on the validity of the local approximation for the self-energy, we may set aside the fact that the Hubbard model at half-filling is expected to be an antiferromagnet 45. Throughout the paper we exclusively consider the (possibly metastable) paramagnetic phase.

The next section briefly introduces the theoretical concept. In section III we present and discuss the results. All calculations have been performed for zero temperature $T=0$. We have limited our investigations to the simple-cubic (sc) lattice. In most cases the (100) surface was considered, but also the more open sc(110) and $\operatorname{sc}(111)$ surfaces were taken into account for comparison. A summary and the conclusions are given in section IV.

\section{THEORY AND CALCULATIONS}

We consider interacting electrons moving through a semi-infinite lattice. The dynamics of the system is described by the Hubbard model. Using standard notations the Hamiltonian reads:

$$
H=\sum_{\langle i j\rangle \sigma}\left(T_{i j}-\mu \delta_{i j}\right) c_{i \sigma}^{\dagger} c_{j \sigma}+\frac{1}{2} U \sum_{i \sigma} n_{i \sigma} n_{i-\sigma}
$$

The system geometry is implicit: $c^{\dagger}(c)$ is the creation (annihilation) operator for a valence electron. The lower indices specify the orbital of the one-particle basis. $\sigma=\uparrow$ ,$\downarrow$ accounts for the spin direction, and $i$ and $j$ refer to the sites within the semi-infinite lattice. We consider the system to be built up from single two-dimensional layers parallel to the surface. Accordingly, the site index may be decomposed as $i=\left(i_{\perp}, i_{\|}\right)$where $i_{\perp}$ labels the layers $i_{\perp}=1, \ldots, \infty$ and $i_{\|}$the sites within a given layer. $i_{\perp}=1$ stands for the topmost surface layer. Within a tight-binding approach the hopping integrals $T_{i j}$ are assumed to be non-zero up to nearest neighbors only and constant throughout the system, i. e.: $T_{i j}=-t$ for nearest neighbors $i, j$. The energy scale is fixed by taking $t=1$. The energy zero will be chosen such that $T_{i i} \equiv T_{0}=0$. $U$ denotes the on-site Coulomb interaction which is assumed to be layer independent. A change of $U$ as well as of $T_{i j}$ in the vicinity of the surface should be expected for any real system [46]. Here the constancy of the parameters is a model assumption which helps to focus on the most important geometrical aspect, namely the reduction of the coordination number at the surface. Finally, in Eq. (11) $n_{i \sigma}=c_{i \sigma}^{\dagger} c_{i \sigma}$ is the local occupationnumber operator, and $\mu$ denotes the chemical potential.

All properties of the system that we are interested in can be obtained from the one-electron Green function:

$$
G_{i j \sigma}(E)=\left\langle\left\langle c_{i \sigma} ; c_{j \sigma}^{\dagger}\right\rangle\right\rangle_{E} .
$$

Let us assume the one-particle basis orbitals to be real. We then get the usual diagonal $i=j$ as well as the offdiagonal $i \neq j$ quasi-particle density of states (QDOS) via:

$$
\rho_{i j \sigma}(E)=-\frac{1}{\pi} \operatorname{Im} G_{i j \sigma}\left(E+i 0^{+}\right) .
$$

For the semi-infinite system the QDOS will be layer dependent in the vicinity of the surface. The Green function obeys an equation of motion which in real-space representation reads:

$E G_{i j \sigma}(E)=\hbar \delta_{i j}+\sum_{k}\left(T_{i k}-\mu \delta_{i k}+\Sigma_{i k \sigma}(E)\right) G_{k j \sigma}(E)$.

Here we have introduced the electronic self-energy $\Sigma$ which includes all effects of electron correlations.

Within the usual perturbative approach 47 the selfenergy is expanded in powers of the interaction $U$. The first non-trivial term is proportional to $U^{2}$. Using the real-space notation again, we have:

$$
\Sigma_{i j \sigma}(E)=U\left\langle n_{i-\sigma}\right\rangle \delta_{i j}+U^{2} \Sigma_{i j \sigma}^{(2)}(E) .
$$

The term linear in $U$ is the Hartree-Fock self-energy. Restricting ourselves to zero temperature, the secondorder contribution reads:

$$
\begin{aligned}
& \Sigma_{i j \sigma}^{(2)}(E)=\frac{1}{\hbar^{3}} \iiint \frac{\rho_{i j \sigma}^{(0)}(x) \rho_{j i-\sigma}^{(0)}(y) \rho_{i j-\sigma}^{(0)}(z)}{E-x+y-z} \times \\
&(\Theta(-x) \Theta(y) \Theta(-z)+\Theta(x) \Theta(-y) \Theta(z)) d x d y d z .
\end{aligned}
$$

$\Theta$ denotes the step function. Within plain SOPT $\rho^{(0)}$ is the free (off-diagonal) density of states:

$$
\rho_{i j \sigma}^{(0)}(E)=-\left.\frac{1}{\pi} \operatorname{Im} G_{i j \sigma}\left(E+i 0^{+}\right)\right|_{U=0} .
$$

The SOPT-HF approach (cf. e. g. Refs. 17, 33) replaces the free by the Hartree-Fock density of states $\rho^{(1)}$ in the expression (6):

$$
\rho_{i j \sigma}^{(1)}(E)=-\frac{1}{\pi} \operatorname{Im} G_{i j \sigma}^{(1)}\left(E+i 0^{+}\right),
$$

where the HF Green function $G^{(1)}$ has to be calculated from 


$$
\begin{aligned}
& E G_{i j \sigma}^{(1)}(E)=\hbar \delta_{i j} \\
& \quad+\sum_{k}\left(T_{i k}+\left(U\left\langle n_{i-\sigma}\right\rangle-\mu\right) \delta_{i k}\right) G_{k j \sigma}^{(1)}(E) .
\end{aligned}
$$

The ground-state expectation value of the local particle number is obtained by integrating the (fully interacting) QDOS:

$$
\left\langle n_{i \sigma}\right\rangle=\frac{1}{\hbar} \int_{-\infty}^{0} \rho_{i i \sigma}(E) d E .
$$

Eqs. (3) - (10) constitute a closed set of equations for the second-order perturbation theory around the Hartree-Fock solution. Generally, the local particle numbers $\left\langle n_{i \sigma}\right\rangle$ have to be determined self-consistently. Due to the presence of the surface, the self-consistent values are expected to be layer dependent, i. e. dependent on the distance from the surface. A self-consistent solution is not necessary in two cases, namely for an infinitesimally small interaction $U=0^{+}$and for the symmetric case of a paramagnet at half-filling. In the latter, we have $\left\langle n_{i \sigma}\right\rangle=\left\langle n_{i-\sigma}\right\rangle=0.5$ for all particle numbers, and the chemical potential is given by $\mu=U / 2$. (In the following the spin index will be omitted as far as possible; throughout the paper only paramagnetism is considered).

There are two main problems associated with the numerical solution of the SOPT-HF equations. The first one consists in the non-locality of the second-order term of the self-energy (6). For the calculation of the local contribution $i=j$ a three-dimensional energy integral has to be performed. Considering the imaginary part of the self-energy $\operatorname{Im} \Sigma_{i i}^{(2)}\left(E+i 0^{+}\right)$, this reduces to convolution integrals which can be calculated efficiently by applying a Laplace transform. The real part is then obtained through the Kramers-Kronig relation. Details of this procedure can be found e. g. in Refs. [17, 32]. Neglecting all off-diagonal elements in (6) corresponds to the local approximation for the self-energy. The numerical effort needed to account for the complete non-locality of the self-energy can be estimated in the following way: Let us consider the neighbor shells around the site $i$. If there was perfect translational invariance with respect to all three spatial dimensions, symmetry reasons would require all non-local terms $\Sigma_{i j}$ to be equal for sites $j$ belonging to the same shell around the site $i$. Therefore, compared with the local approximation the effort rises by a factor $s+1$ that is determined by the number of shells $s$ that are necessary to obtain convergence for the results 17] (as mentioned in the introduction, the necessary number of shells strongly depends on the lattice dimension $D$ ). In the semi-infinite system the symmetry is reduced, i. e. not all the sites $j$ within the same shell are equivalent. Consider, for example, a (fixed) site $i$ near the surface. Independent from the lattice type and the surface there are three inequivalent possibilities at least to choose a site $j$ within the nearest-neighbor shell of $i$ : $j$ within the same layer as $i\left(j_{\perp}=i_{\perp}\right), j$ within a layer more distant from the surface $\left(j_{\perp}=i_{\perp}+n\right.$ with $\left.n>0\right)$, and finally $j$ closer to the surface $\left(j_{\perp}=i_{\perp}-n\right)$. (Note that nearest neighbors need not necessarily belong to adjacent layers.) Therefore, the reduced symmetry implies an increase in the numerical effort by a factor three or even more, since the number of inequivalent sites $j$ within the same shell around $i$ increases with increasing shell radius. Finally, we have to consider all inequivalent positions for the site $i$. Assuming perfect translational symmetry within the layers parallel to the surface, their number is given by the number of inequivalent layers $N_{\perp}$. The computational effort therefore rises once more by a factor $N_{\perp}$. With increasing distance of $i$ and $j$ to the surface, $\Sigma_{i j}(E)$ converges to the bulk self-energy. Thus $N_{\perp}$ can be regarded to be finite but may be rather large (several tens of layers). This also implies that instead of the semi-infinite we can assume a slab geometry with a large number of layers $d\left(\sim 2 N_{\perp}\right)$ in the actual calculation.

The second main problem concerns the equation of motion (4) for the Green function. It is the reduced translational symmetry of the semi-infinite system again that renders its solution more difficult. In order to exploit translational symmetry within the layers, twodimensional Fourier transformation of any quantity $F_{i j}$ will be convenient:

$$
F_{i j} \equiv F_{i_{\|} j_{\|}}^{i_{\perp} j_{\perp}}=\frac{1}{N_{\|}} \sum_{\mathbf{k}_{\|}} e^{i \mathbf{k}_{\|}\left(\mathbf{R}_{i_{\|}}-\mathbf{R}_{j_{\|}}\right)} F_{i_{\perp} j_{\perp}}\left(\mathbf{k}_{\|}\right),
$$

and

$$
F_{i_{\perp} j_{\perp}}\left(\mathbf{k}_{\|}\right)=\frac{1}{N_{\|}} \sum_{i_{\|} j_{\|}} e^{-i \mathbf{k}_{\|}\left(\mathbf{R}_{i \|}-\mathbf{R}_{j_{\|}}\right)} F_{i_{\|} j_{\|}}^{i_{\perp} j_{\perp}} .
$$

Here $\mathbf{k}_{\|}$is a wave vector of the first two-dimensional surface Brillouin zone. After Fourier transformation the equation of motion for the Green function reads:

$$
\begin{aligned}
& E G_{i_{\perp} j_{\perp}}\left(\mathbf{k}_{\|}, E\right)=\hbar \delta_{i_{\perp} j_{\perp}}+\sum_{k_{\perp}}\left(T_{i_{\perp} k_{\perp}}\left(\mathbf{k}_{\|}\right)\right. \\
& \left.-\mu \delta_{i_{\perp} k_{\perp}}+\Sigma_{i_{\perp} k_{\perp}}\left(\mathbf{k}_{\|}, E\right)\right) G_{k_{\perp} j_{\perp}}\left(\mathbf{k}_{\|}, E\right) .
\end{aligned}
$$

This may be considered as a matrix equation for $G_{i_{\perp} j_{\perp}}$. The dimension of the inversion problem is given by the slab thickness $d \sim 2 N_{\perp}$. Within the tight-binding approach, the hopping matrix $T_{i_{\perp} j_{\perp}}$ as well as the selfenergy matrix $\Sigma_{i_{\perp} j_{\perp}}$ are sparse. Therefore, the tightbinding recursion technique 48, 49, 21] is most suitable for its solution. The application of the method yields the Green function as a continued fraction:

$$
\begin{aligned}
& G_{i_{\perp} i_{\perp}}\left(\mathbf{k}_{\|}, E\right) \equiv G_{i_{\perp} i_{\perp}}=G_{i_{\perp}}^{(0)} \\
& G_{i_{\perp}}^{(k)}=\frac{\hbar}{E-a_{i_{\perp}}^{(k)}-\left(b_{i_{\perp}}^{(k+1)}\right)^{2} G_{i_{\perp}}^{(k+1)}} .
\end{aligned}
$$


The (energy-dependent) coefficients $a_{i_{\perp}}^{(k)}$ and $b_{i_{\perp}}^{(k+1)}$ can be calculated iteratively within the recursion approach. Due to the finite dimensionality, the continued fraction terminates automatically: $k=0, \ldots d-1$. Off-diagonal elements of the Green function can be obtained by means of a polarization relation [21].

\section{RESULTS}

We have chosen the simple-cubic (sc) lattice as a model system for our calculations. Three different lowindex surfaces, sc(100), sc(110) and sc(111), have been considered. Let us start the discussion of the results with the (100) face. For the sc lattice the nearest-neighbor coordination number for sites in the bulk is $z=6$. Speaking in terms of the (100) layers parallel to the surface, each site has $z_{\|}^{(100)}=4$ nearest neighbors within the same layer and $z_{\perp}^{(100)}=2$ sites in the adjacent layers. For the top-layer sites $\left(i_{\perp}=1\right)$ the coordination number is thus reduced to $z_{S}^{(100)}=5$.

This (small) lessening of the coordination has consequences for the free $(U=0)$ density of states (DOS) $\rho_{i j}^{(0)}(E)$. The free DOS determines via Eq. 6 the secondorder contribution of the self-energy for $U=0^{+}$. For finite $U$ and at half-filling we have to consider the HartreeFock $(\mathrm{HF}) \operatorname{DOS} \rho_{i j}^{(1)}(E)$. Compared with the free DOS the HF-DOS is only shifted constantly in energy by the amount $U\left\langle n_{i-\sigma}\right\rangle=U / 2$ which is compensated by the corresponding shift of the chemical potential. So in both cases $\rho_{i j}^{(0)}(E)$ is the primary quantity of interest.

Let us discuss first the layer-dependent diagonal elements $(i=j)$ of the free DOS which are shown in Fig. 1. We notice that there are considerable differences between the DOS of the topmost surface layer and the bulk DOS. The difference becomes weaker and weaker with increasing distance from the surface. The surface can be regarded as a perturbation of the infinitely extended lattice that gives rise to oscillations of the layerdependent charge density. These surface Friedel oscillations [50] are closely related to the periodic deformations of the DOS which, as can be seen in Fig. 1, are damped when passing from the very surface to the bulk. The layer-dependent off-diagonal DOS $\rho_{i j}^{(0)}(E)$ for nearestneighbors $i$ and $j$ within the same layer is also shown in Fig. 1. Its absolute magnitude is smaller, and there is a zero of the DOS at the center of gravity $T_{0}=0$ of the diagonal DOS. Compared with the case $i=j$, we observe qualitatively similar differences between the top-layer DOS and the bulk DOS as well as the signature of surface Friedel oscillations.

The overall shape of the free DOS in the vicinity of the surface can be understood in terms of the moments of the DOS. These are defined as:

$$
M_{i j}^{(n, 0)}=\int_{-\infty}^{\infty} E^{n} \rho_{i j}^{(0)}(E) d E .
$$

An alternative, but equivalent representation can be de-

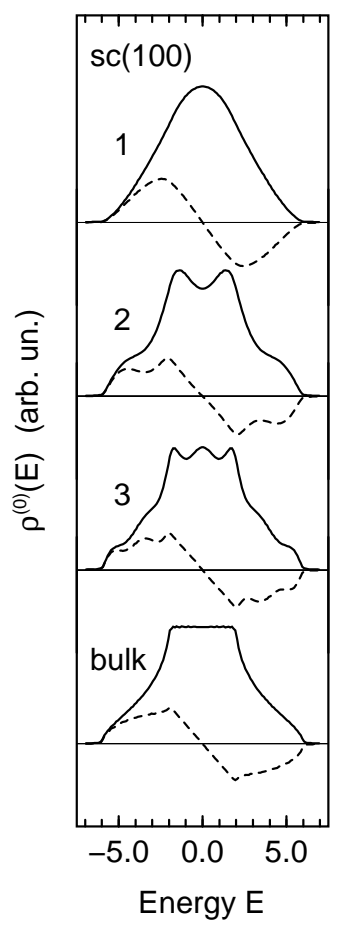

Fig. 1. Free $(U=0)$ densities of states $\rho_{i i}^{(0)}$ for the first three layers from the surface $\left(i_{\perp}=1-3\right)$ and free bulk density of states $\left(i_{\perp}=\infty\right)$ as functions of energy (solid lines). $i_{\perp}=1$ stands for the outermost surface layer. Dashed lines: offdiagonal free densities of states $\rho_{i j}^{(0)}(E)$ for nearest-neighbor sites $i$ and $j$, both within the same (indicated) layer. Calculations for the $\operatorname{sc}(100)$ surface. (A slab thickness of $d=50$ turns out to be sufficient for convergence in all calculations). All energies are given in terms of the nearest-neighbor hopping $t=1 . \mu=0$.

rived from the equation of motion for the free Green function:

$$
M_{i j}^{(n, 0)}=\sum_{i_{1} \cdots i_{n-1}} T_{i i_{1}} T_{i_{1} i_{2}} \cdots T_{i_{n-1} j} .
$$

Using this equation we can conclude that for each layer the diagonal $(i=j)$ DOS must be a symmetric function of energy with respect to its center of gravity $T_{0}=0$ : All odd moments must vanish since there is no way to start from and to return to a site $i$ by an odd number of nearest-neighbor hoppings for a (semi-infinite) simple cubic lattice. Analogously, the off-diagonal free DOS for nearest-neighbors $i$ and $j$ must be antisymmetric.

Comparing with the bulk, the on-site top-layer DOS is much more compressed. This "band-narrowing" is a consequence of the reduced coordination number of the top-layer sites and can be understood by considering the second moment:

$$
\Delta^{2} \rho_{i i}^{(0)}=M_{i i}^{(2,0)}-\left(M_{i i}^{(1,0)}\right)^{2}=\sum_{j \neq i} T_{i j}^{2}=z(i) t^{2} .
$$

The reduced coordination number $z(i)$ of the site $i$ at the surface yields a reduced variance $\Delta^{2} \rho^{(0)}$ of the top- 


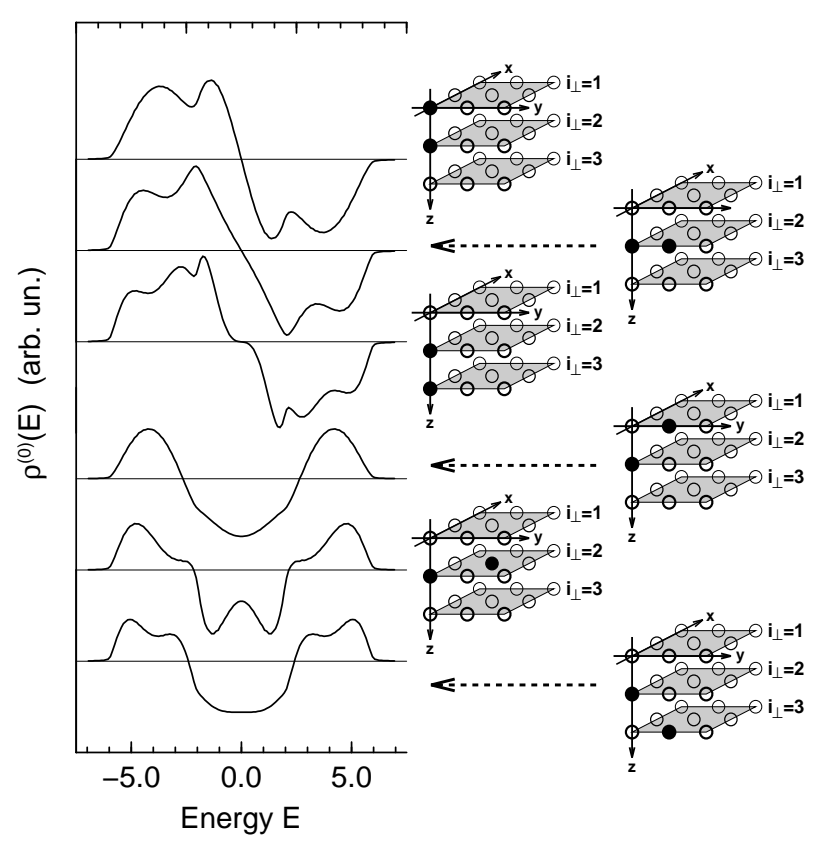

Fig. 2. Free off-diagonal densities of states $\rho_{i j}^{(0)}(E)$ near the $\operatorname{sc}(100)$ surface. The respective sites $i$ and $j$ are indicated as filled circles in the corresponding schematic drawing of the surface geometry. The shaded planes represent the first three layers from the surface, $i_{\perp}=1-3\left(i_{\perp}=1\right.$ : top surface layer). Note that $\rho_{i j}^{(0)}(E)=\rho_{j i}^{(0)}(E) . \mu=0$.

layer DOS. For the second and the third surface layer higher moments have to be considered. Despite the band-narrowing effect, the densities of states of all layers share common band edges. For a spatially constant hopping parameter $t$ no split-off states can be observed.

The difference between the free densities of states for non-equivalent pairs of sites $i$ and $j$ is demonstrated in Fig. 2. For the calculations the site $i$ has been kept fixed within the second layer from the surface $\left(i_{\perp}=2\right)$. The first three panels from the top in Fig. 2 show the off-diagonal DOS $\rho_{i j}^{(0)}(E)$ for nearest-neighbor sites $j$. There are three non-equivalent positions relative to $i$. We recognize significant though not strong differences between the spectra. These are exclusively due to the presence of the surface; choosing for $i$ a site within the bulk would yield identical results in all three cases. The last three panels show the DOS for $i$ within the second layer as before, but $j$ a next-nearest neighbor. Again, three non-equivalent positions for $j$ can be found. Since an even number of nearest-neighbor hoppings is needed to get from site $i$ to site $j$, the DOS is symmetric. The differences between the spectra are non-negligible and of the same order of magnitude as in the nearest-neighbor case. They will lead to a (weak) directional dependence of the self-energy in the vicinity of the surface.

The local approximation for the self-energy $\Sigma_{i j}(E)$ can only be a reasonable starting point if the non-local parts strongly decrease with increasing distance between the sites $i$ and $j$. Within the perturbational approach

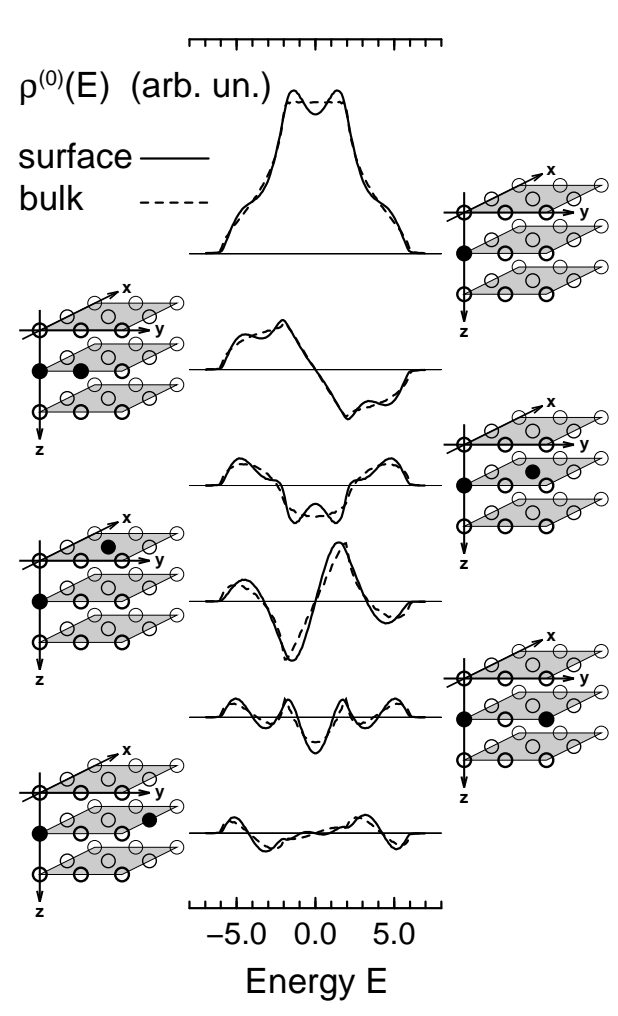

Fig. 3. Free densities of states $\rho_{i j}^{(0)}(E)$ near the sc(100) surface (solid lines). Sites $i$ and $j$ are indicated (shaded planes: (100) layers, top plane: surface layer). Dashed lines: bulk densities of states. Relative position of sites $i$ and $j$ as indicated (shaded planes now to be interpreted as (100) layers in the bulk). $\mu=0$.

these are determined by the off-diagonal free DOS. In Fig. 3 the $\operatorname{DOS} \rho_{i j}^{(0)}(E)$ is shown for a site $i$ fixed within the second layer from the surface and different sites $j$ with an increasing distance $d_{i j} \equiv\left\|\mathbf{R}_{i}-\mathbf{R}_{j}\right\|$ (from the top). It can be seen that the absolute magnitude of the DOS decreases with increasing distance $d_{i j}$ indeed. Particularly, the off-diagonal DOS for nearest neighbors is clearly smaller in absolute values compared with the onsite DOS over the whole energy range. A more quantitative estimate can be given by integrating the absolute DOS. We then obtain the ratio (for increasing distance, on-site DOS normalized to 100) $100: 33: 21: 28: 14: 9$. The decrease is not monotonous. According to Eq. (6) a stronger decrease has to be expected for the $U^{2}$ contribution to the self-energy since $\Sigma_{i j}^{(2)}$ (roughly) results from the third power of the DOS $\left(\rho_{i j}^{(0)}\right)^{3}$.

The results for the site $i$ located near the surface can be compared with the results for a site $i$ in the bulk which are also shown in Fig. 3. The differences between the corresponding surface and bulk off-diagonal densities of states are significant though not strong. They are qualitatively similar to the difference between the surface and bulk on-site DOS. Since the second-order contribution to the self-energy (6) results from a (two- 


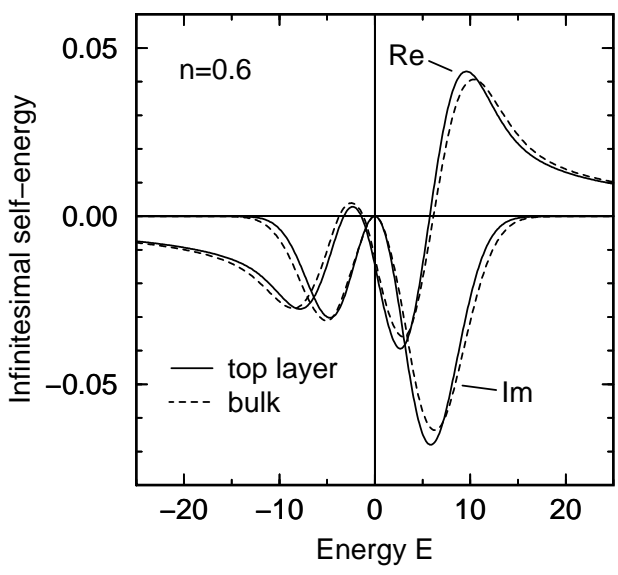

Fig. 4. Real and imaginary part of the infinitesimal on-site self-energy $\left.\frac{1}{2} \frac{d^{2}}{d U^{2}} \Sigma_{i i}\left(E+i 0^{+}\right)\right|_{U=0}$ of the semi-infinite $\operatorname{sc}(100)$ Hubbard model. Band-filling: $n=0.6$. Solid line: site $i$ within the topmost surface layer $\left(i_{\perp}=1\right)$. Dashed line: bulk self-energy.

fold) convolution of the free DOS, these differences become more or less meaningless. We thus can conclude that the self-energy $\Sigma_{i j}^{(2)}(E)$ for a site $i$ within second layer and for an arbitrary site $j$ is almost identical to the corresponding diagonal or off-diagonal self-energy in the bulk. Therefore, for a site $i$ in the second surface layer the local approximation is likewise appropriate (or inappropriate) as for a site $i$ in the bulk.

Let us consider now the top layer where the situation is found to be different. Fig. 4 shows numerical results for the infinitesimal on-site self-energy $\left.\frac{1}{2} \frac{d^{2}}{d U^{2}} \Sigma_{i i}(E)\right|_{U=0}$ of the semi-infinite sc(100) Hubbard model. We consider a non-symmetric case: The chemical potential has been chosen such that in the bulk the average occupation number (band-filling) is $n=2\left\langle n_{i \sigma}\right\rangle=0.6$. It can be seen in Fig. 4 that there are significant differences between the top-layer and the bulk self-energy. Contrary, the infinitesimal on-site self-energy of the second layer is almost indistinguishable from the bulk self-energy. On the energy scale used in the figure, differences would not be visible. The same holds true for the third and all other surface layers.

Fig. 4 shows that the on-site self-energy of the top layer is more compressed compared with the bulk selfenergy; the effective width of the imaginary part is smaller at the surface. This is a typical surface effect since it is a direct consequence of the narrowing of the top-layer DOS mentioned above. Another consequence concerns the local occupation number $\left\langle n_{i \sigma}\right\rangle$ at the surface. Due to the narrowing of the top-layer DOS, the surface charge density $n_{\mathrm{s}} \equiv 2\left\langle n_{i \sigma}\right\rangle$ (for $i$ within the first layer) must be smaller than the bulk band-filling: $n_{\mathrm{s}}<n$ (below half-filling) 20]. For $n=0.60$ we find $n_{\mathrm{s}}=0.56$. This leads to a reduced total weight $w_{\mathrm{s}}$ of the imaginary part of the infinitesimal self-energy at the surface compared with its weight in the bulk $w$ : From Eq. (6) we have: $w_{(\mathrm{s})} \equiv-\frac{1}{\pi} \operatorname{Im} \int_{-\infty}^{\infty} \Sigma_{i i}^{(2)}\left(E+i 0^{+}\right) d E=\frac{n_{(\mathrm{s})}}{2}\left(1-\frac{n_{(\mathrm{s})}}{2}\right)$.

The difference is found to be small: $w=0.21$ and $w_{\mathrm{s}}=$ 0.20 .

Both, the imaginary part of the top-layer and of the bulk self-energy, vanish quadratically, $-\operatorname{Im} \Sigma_{i i}^{(2)}(E) \sim$ $E^{2}$, as $E \mapsto 0$. This follows from a straightforward analysis of Eq. (6) [51 and implies the existence of a well-defined Fermi surface [52]. We can thus distinguish between the damping of the occupied $(E<0)$ and of the unoccupied $(E>0)$ part in the excitation spectrum. Let $w_{(\mathrm{s})}^{<}$and $w_{(\mathrm{s})}^{>}$denote the integrated weight of $-\frac{1}{\pi} \operatorname{Im} \Sigma_{i i}^{(2)}\left(E+i 0^{+}\right)$at the surface or in the bulk for $E<0$ and $E>0$, respectively. We have $w_{(\mathrm{s})}^{<}+w_{(\mathrm{s})}^{>}=w_{(\mathrm{s})}$, and from Eq. (6) the following result can be derived:

$$
\begin{aligned}
& w_{(\mathrm{s})}^{<}=\left(\frac{n_{(\mathrm{s})}}{2}\right)^{2}\left(1-\frac{n_{(\mathrm{s})}}{2}\right), \\
& w_{(\mathrm{s})}^{>}=\frac{n_{(\mathrm{s})}}{2}\left(1-\frac{n_{(\mathrm{s})}}{2}\right)^{2} .
\end{aligned}
$$

It can be seen in Fig. 4 that for the occupied part the integrated weight is clearly smaller in the top layer. This has to be interpreted as another typical surface effect: The reduction of the coordination number at the surface implies the narrowing of the top-layer DOS and thus the lowered charge density; via Eq. (19) this leads to a smaller damping in the occupied part. We get $w_{\mathrm{s}}^{<}=$ 0.056 to be compared with the bulk value $w^{<}=0.063$. On the contrary, the weight within the unoccupied part is almost unchanged: $w_{\mathrm{s}}^{>}=0.145$ and $w^{>}=0.147$.

The non-local parts of the infinitesimal self-energy for the top layer of the sc(100) surface and for the bulk are shown in Fig. 5. Unlike the imaginary part of onsite (retarded) self-energy, which must be negative over the whole energy range, there is no definite sign for the imaginary part in the off-diagonal case. Instead, we have the sum rule $\int_{-\infty}^{\infty} \operatorname{Im} \Sigma_{i j}^{(2)}\left(E+i 0^{+}\right) d E=0$ for $i \neq j$. The low-energy behavior, however, remains unchanged: the imaginary part vanishes quadratically for $E \mapsto 0$, i. e. $\operatorname{Im} \Sigma_{i j}^{(2)}(E) \sim \pm E^{2}$ for $i \neq j$. The sign depends on the sign of the (off-diagonal) DOS at the Fermi edge. This can be verified by expanding the infinitesimal selfenergy in powers of $E$. Similar to the analysis in Ref. 51], one may derive the following result in the real-space representation:

$$
-\frac{1}{\pi} \operatorname{Im} \Sigma_{i j}^{(2)}\left(E+i 0^{+}\right)=\left(\frac{1}{\hbar} \rho_{i j}^{(0)}(0)\right)^{3} \times E^{2}+\mathcal{O}\left(E^{4}\right) .
$$

Consider, for example, nearest-neighbor sites $i$ and $j$ and $n<1$. As can be seen from Fig. $1, \rho_{i j}^{(0)}(E)$ is positive at the Fermi energy and thus $\operatorname{Im} \Sigma_{i j}^{(2)}\left(E+i 0^{+}\right) \sim-E^{2}$ 


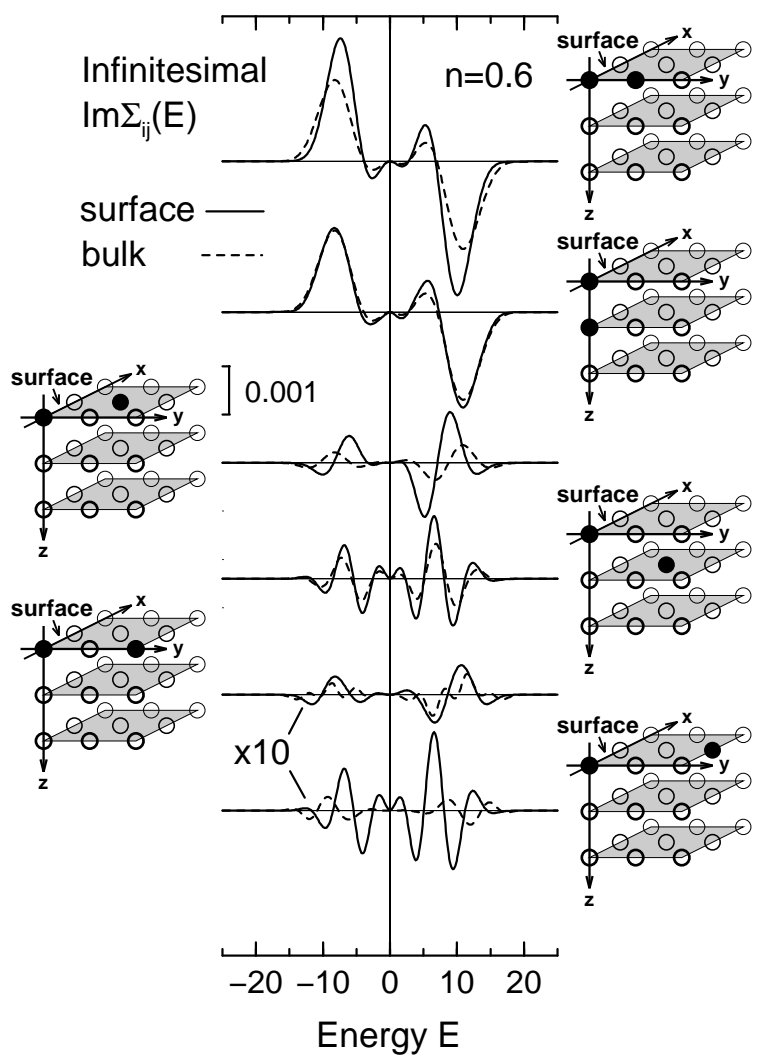

Fig. 5. Off-diagonal elements of the imaginary part of the infinitesimal self-energy $\left.\frac{1}{2} \frac{d^{2}}{d U^{2}} \Sigma_{i j}\left(E+i 0^{+}\right)\right|_{U=0}$ near the $\operatorname{sc}(100)$ surface (solid lines) and in the bulk (dashed lines). Band-filling $n=0.6$. Sites $i \neq j$ as indicated (dashed lines/bulk: shaded planes represent (100) bulk layers). The vertical energy scale is given by the height of the bar $(0.001$ in units of $t$ ). In the last two panels the data have been multiplied by a factor 10 .

for $E \mapsto 0$ which is consistent with the result shown in Fig. 5 .

The first two panels from the top in Fig. 5 show the imaginary part of the self-energy for two non-equivalent pairs of nearest-neighbor sites $i$ and $j$. A significantly stronger variation of the self-energy is observed when both sites are located within the top layer. This implies a directional dependence of the self-energy which, however, is confined to the very surface. In the bulk both cases are equivalent, and the same result for the selfenergy is found. Generally we can state that significant though not strong differences to the bulk self-energy are only found in those cases where both sites $i$ and $j$ belong to the topmost surface layer. This again proves that the presence of the surface manifests itself in the self-energy for the top surface layer only.

Increasing the distance $d_{i j}$ between the sites, results in a rapid decrease of the absolute values for the selfenergy in the bulk as well as in the vicinity of the surface. Compared with the on-site self-energy (Fig. 4), the absolute values of the non-local self-energy are smaller by more than one order of magnitude already for nearest-

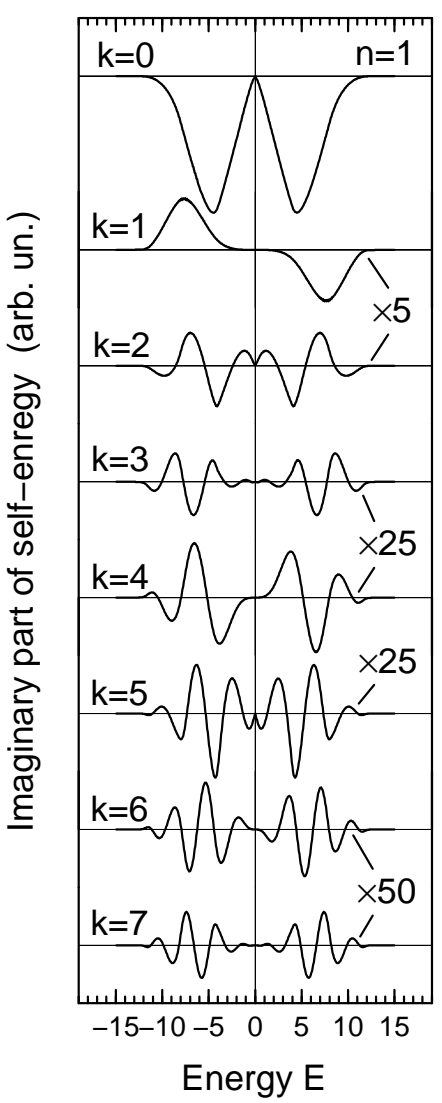

Fig. 6. Imaginary part of the (infinitesimal) self-energy $\left.\frac{1}{2} \frac{d^{2}}{d U^{2}} \Sigma_{i j}\left(E+i 0^{+}\right)\right|_{U=0}$ for the two-dimensional $(D=2)$ Hubbard model on the square lattice at half-filling $(n=1)$. Site $j$ within the $k$-th neighbor shell of the (fixed) site $i$. Results for $k=0$ (on-site self-energy) up to $k=7$. The results for $k \neq 0$ have been scaled as indicated.

neighbor sites $i$ and $j$. We have integrated the energydependent expression $\left|\operatorname{Im} \Sigma_{i j}^{(2)}\left(E+i 0^{+}\right)\right|$for $i=j$ (Fig. 4) and for $i \neq j$ (second to sixth panel in Fig. 5) to get a more quantitative estimate for the magnitude of the different contributions at the very surface. Normalizing the local contribution to 100 , the following ratio is obtained for increasing distance: $100: 4.3: 1.4: 1.6: 0.09: 0.20$. As had to be expected, this roughly corresponds to the above-mentioned ratio for the third powers of the integrated (absolute) densities of states. It shows up that the local part of the self-energy (bulk as well as surface) is rather dominating.

The results for the semi-infinite lattice may be compared with those for a two-dimensional case. For this purpose we have calculated the local and the non-local parts of the infinitesimal self-energy on the $D=2$ square lattice. Fig. 6 shows the results for the imaginary part at half-filling. The self-energy $\Sigma_{i j}^{(2)}(E)$ is a symmetric (antisymmetric) function of energy with respect to $E=0$ if an even (odd) number of nearest-neighbor hoppings is necessary to get from site $i$ to site $j$. At half-filling the low-energy behavior of the self-energy is likewise excep- 


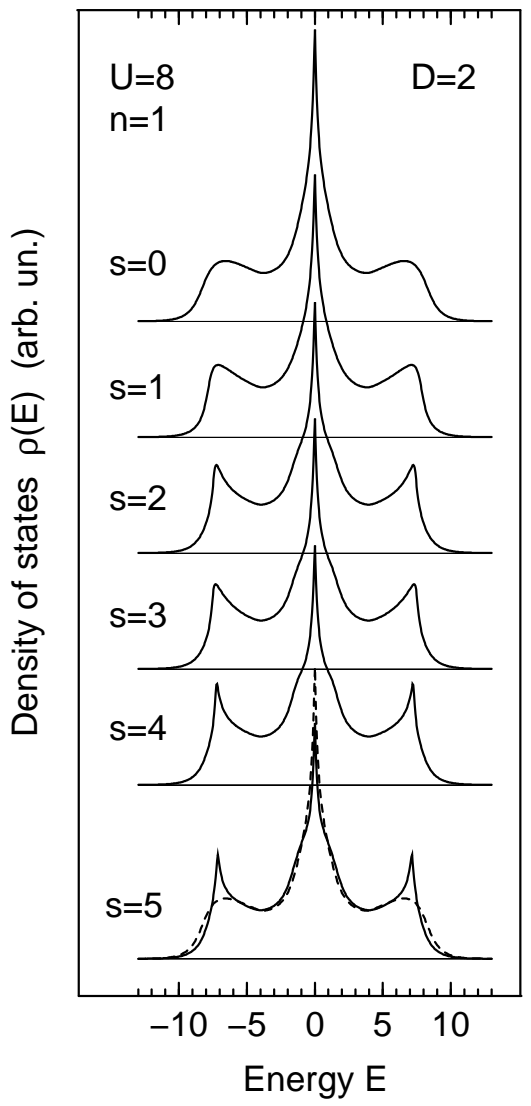

Fig. 7. Quasi-particle density of states $\rho(E)$ of the $D=2$ Hubbard model on the square lattice at half-filling and $U=$ 8 within SOPT-HF. Off-diagonal contributions of the selfenergy included up to the s-th neighbor shell. Results for $s=0$ (local approximation) up to $s=5$. Dashed line: $s=0$ result for comparison.

tional: Expanding the expression (6) for small $E$ yields for the on-site part: $-\frac{1}{\pi} \operatorname{Im} \Sigma_{i i}^{(2)}\left(E+i 0^{+}\right) \sim E^{2}|\ln | E \|^{3}$ as $E \mapsto 0$ [53]. The reason for this anomalous behavior at half-filling is the logarithmic divergence of the free DOS at $E=0$ in two dimensions.

As can be seen from Fig. 6, the non-local parts of the self-energy are much smaller in absolute magnitude compared with the local one. Again they are found to decrease with increasing distance $d_{i j}$. For the integrated absolute self-energies we obtain the following ratio: $100: 10: 7.4: 1.1: 2.1: 2.0: 0.80: 0.52$ (onsite part normalized to 100). Compared with the results found for the $\mathrm{sc}(100)$ surface, we notice a considerably slower decrease with increasing distance $d_{i j}$. Qualitatively, this result does not depend on the band-filling at all. Although the mere numbers should not be overemphasized, the comparison of the different ratios surely gives an impression about the relative importance of the non-locality of the self-energy in the different cases.

So far only the free DOS as well as the self-energy have been considered. To be able to judge on the quality of the local approximation, however, one also has to check the effects of the non-local parts on the quasiparticle density of states (QDOS): While $\left|\Sigma_{i j}^{(2)}\left(E+i 0^{+}\right)\right|$

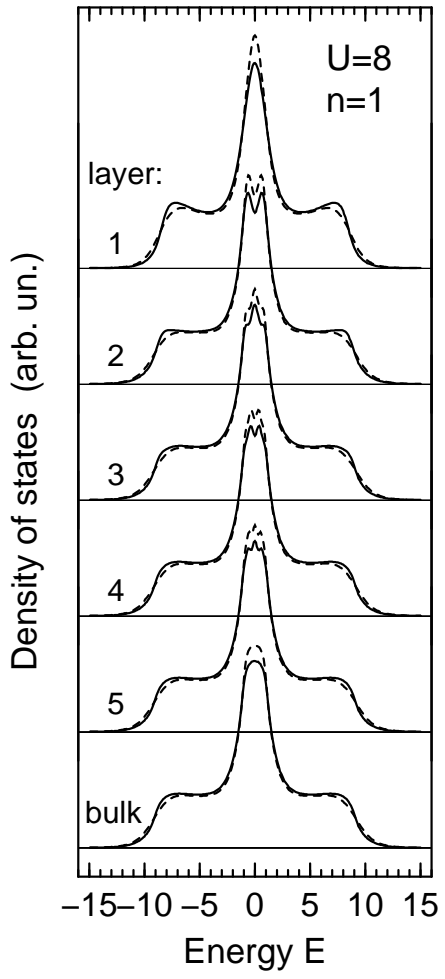

Fig. 8. Layer-dependent quasi-particle density of states $\rho_{i i}(E)$ for the semi-infinite sc(100) Hubbard model at halffilling and $U=8$. SOPT-HF results for the first five layers from the surface and for the bulk. Dashed lines: local approximation. Solid lines: non-locality of the self-energy fully taken into account.

may be a small quantity for a given pair of sites $i \neq j$, it nevertheless may be important with respect to the QDOS provided that a large number of equivalent pairs have to be taken into account. For the discussion of the (paramagnetic) QDOS at finite interaction $U$, we restrict ourselves to the symmetric case of half-filling. As mentioned in the introduction, the SOPT-HF scheme reproduces a large number of exactly solvable limits for $n=1$ and should thus yield reliable results, at least on a qualitative level.

The QDOS of the $D=2$ square lattice at half-filling is shown in Fig. 7. We have chosen $U=8$ which is equal to the free Bloch band width $W$. The first panel shows the result for the local approximation $(s=0)$. The other panels show calculations where we have included the non-local parts of the self-energy up to $s$-th neighbor shell ( $s=1$ to $s=5)$. Qualitatively, all spectra look similar: The high-energy charge excitation peaks (Hubbard bands) are clearly visible for $U=8$, and a quasiparticle resonance remains around $E=0$. Yet there are important differences: The inclusion of more and more shells narrows the whole spectrum, sharpens the chargeexcitation peaks while the resonance looses some spectral weight. Furthermore, a new structure comes into existence at $E \approx \pm 1$. Fully converged results cannot be obtained until $s=5$ [54]. 


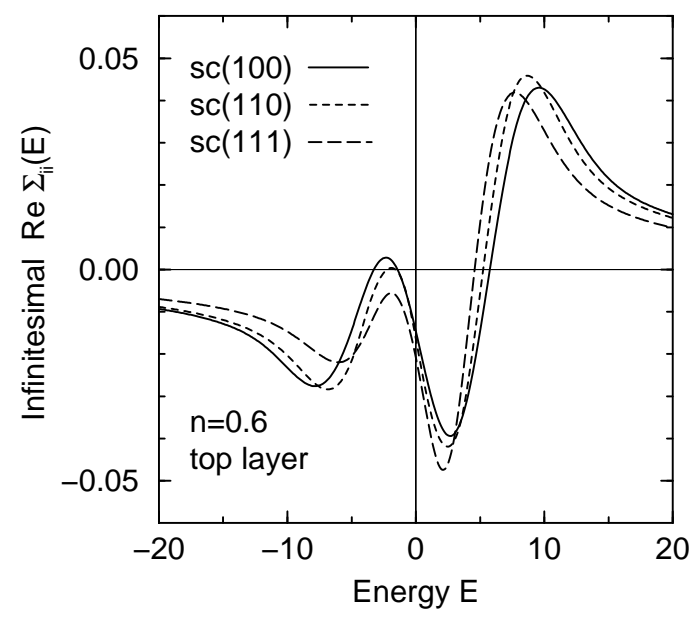

Fig. 9. Real part of the infinitesimal on-site self-energy $\left.\frac{1}{2} \frac{d^{2}}{d U^{2}} \Sigma_{i i}\left(E+i 0^{+}\right)\right|_{U=0}$ for the top surface layer of the semiinfinite Hubbard model. Results for three different surfaces. Band-filling: $n=0.6$.

Let us now return to the $\mathrm{sc}(100)$ surface again. The layer-dependent QDOS in the vicinity of the surface and in the bulk for $U=8$ and at half-filling are shown Fig. 8. For each layer the QDOS exhibits three main features which again can be interpreted as the two chargeexcitation peaks (on the high-energetic sides) and a resonance (at the Fermi level).

The discussion of the self-energy above has already indicated that effects of its non-locality are less important for the semi-infinite system compared with $D=2$. This becomes manifest when considering the QDOS at the surface. Taking into account the on-site self-energy and the nearest-neighbor non-local part is almost sufficient to obtain fully convergent results. Remaining differences between the QDOS for $s=1$ and for higher $s$ would hardly be visible on the scale used in Fig. 8. Let us point out that the convergence with respect to $s$ is equally fast for both, the top-layer and the bulk QDOS 55 .

The results shown in the figure prove that the local approximation provides a reasonable description of all features appearing in the spectra. Only the spectral weight of the resonance at the Fermi edge is somewhat overestimated, and the charge-excitation peaks are less pronounced for $s=0$. Compared with the results for the $D=2$ square lattice, however, the local approximation is only slightly better. In any case it is likewise appropriate for the bulk as well as for the surface including the top layer.

The results for the QDOS at the sc(100) surface are interesting of their own: First, we notice that the top-layer QDOS is slightly narrowed. This can be understood by referring to the moments again. The first few moments of the QDOS,

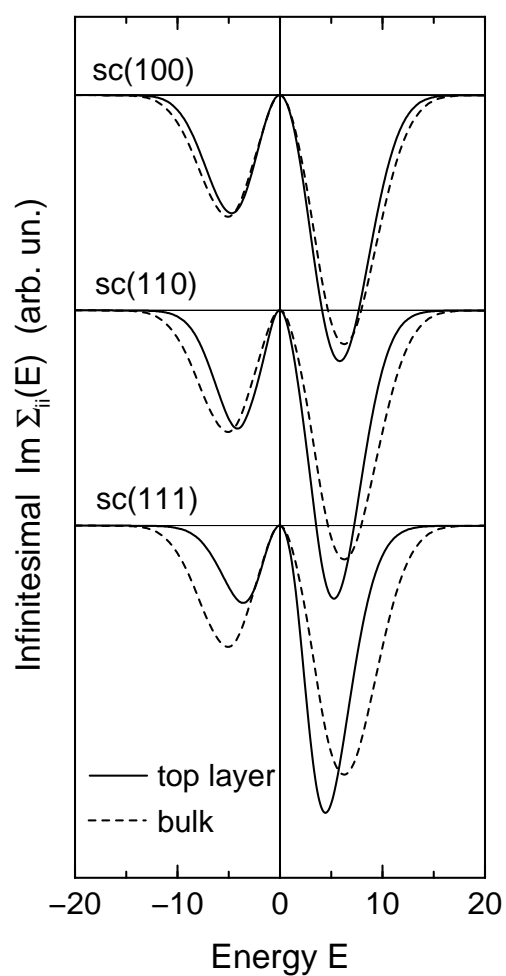

Fig. 10. Imaginary part of the infinitesimal on-site selfenergy $\left.\frac{1}{2} \frac{d^{2}}{d U^{2}} \Sigma_{i i}\left(E+i 0^{+}\right)\right|_{U=0}$ of the semi-infinite Hubbard model at $n=0.6$. Solid lines: site $i$ within the top surface layer. Dashed lines: bulk.

$$
M_{i j}^{(n)}=\int_{-\infty}^{\infty} E^{n} \rho_{i j}(E) d E,
$$

can be calculated exactly (see Ref. 21, for example):

$$
\begin{aligned}
& M_{i i}^{(0)}=1 \\
& M_{i i}^{(1)}=T_{0}+U\left\langle n_{i-\sigma}\right\rangle \\
& M_{i i}^{(2)}=\sum_{j} T_{i j}^{2}+2 U T_{0}\left\langle n_{i-\sigma}\right\rangle+U^{2}\left\langle n_{i-\sigma}\right\rangle .
\end{aligned}
$$

The variance of the QDOS for the layer $i_{\perp}$ is then given by the expression:

$$
\Delta^{2} \rho_{i i}=M_{i i}^{(2)}-\left(M_{i i}^{(1)}\right)^{2}=\sum_{j \neq i} T_{i j}^{2}+U^{2}\left\langle n_{i-\sigma}\right\rangle\left(1-\left\langle n_{i-\sigma}\right\rangle\right) .
$$

We can conclude that increasing $U$ leads to a strong increase of the variance of the QDOS which is mainly due to the evolution of the two Hubbard bands. For a given $U$ and at half-filling the second term on the righthand side of (23) is a constant. As for the case of the free DOS, the first term implies a narrowing of the toplayer QDOS because of the reduced coordination number for sites in the first layer. This can be seen in Fig. 8 . Additionally, we notice a slight narrowing of the quasiparticle resonance for the top layer. 


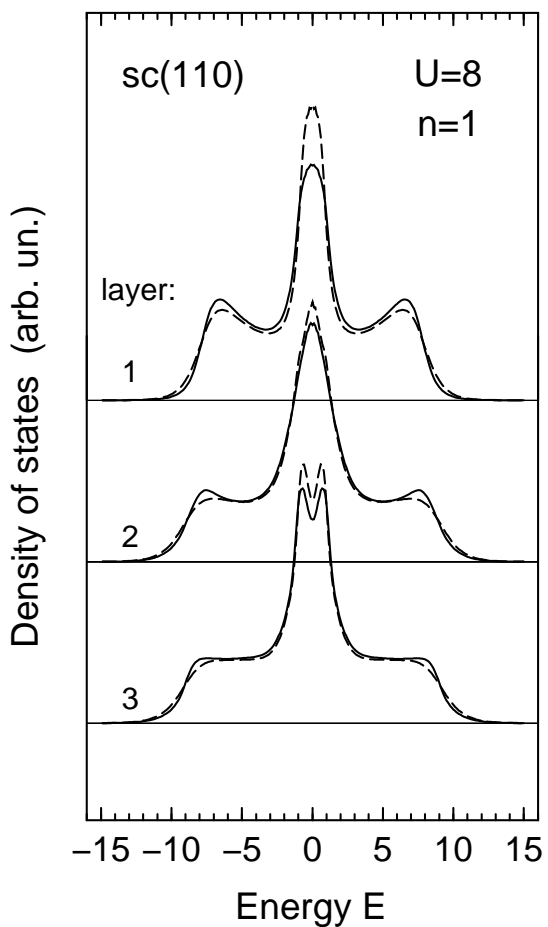

Fig. 11. Layer-dependent quasi-particle density of states for the $\operatorname{sc}(110)$ surface at half-filling and $U=8$. SOPT-HF results for the first three layers from the surface. Dashed lines: local approximation for the self-energy. Solid lines: non-locality of self-energy fully taken into account.

Compared with the free DOS in Fig. 1, the differences between the QDOS for the different layers are much smaller in general. This is a consequence of quasiparticle damping due to the imaginary part of the selfenergy. Generally this indicates a damping of the surface Friedel oscillations due to electron correlations. Note that the effect is also reproduced by the local approximation. The strong periodic deformations of the DOS in the vicinity of the surface that could be observed for the free $(U=0)$ system (Fig. 1$)$, are now confined to the resonance in small energy range around the Fermi energy. Here they appear as tiny wiggles only.

The effects that result from the reduction of the coordination number at the surface show up more clearly when comparing results for different surfaces. For this purpose we also considered the (110) and the (111) surface of the sc lattice. Thinking the respective system to be built up from layers parallel to the surface, each site except for those in the first layer has $z_{\|}^{(110)}=2$ $\left(z_{\|}^{(111)}=0\right)$ nearest neighbors within the same and $z_{\perp}^{(110)}=4\left(z_{\perp}^{(111)}=6\right)$ nearest neighbors in the adjacent layers. This implies $z_{S}^{(110)}=4$ and $z_{S}^{(111)}=3$ for the coordination numbers of the top-layer sites, and we have $z_{S}^{(100)}>z_{S}^{(110)}>z_{S}^{(111)}$.

This sequence can be recovered in the results for the top-layer self-energy for the different surfaces. Fig. 9 shows their real parts for $U=0^{+}$and bulk band-filling

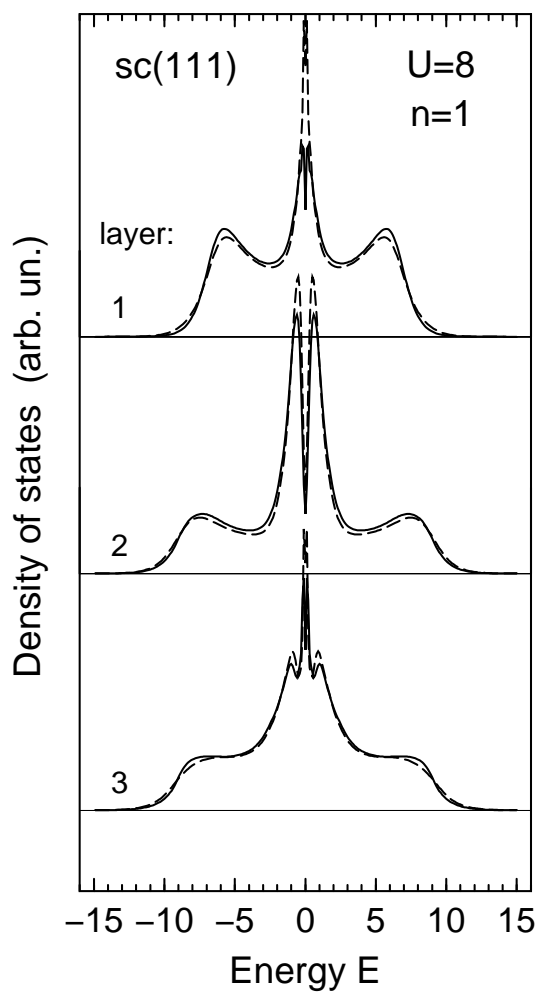

Fig. 12. The same as Fig. 11, but for the sc(111) surface.

$n=0.6$. The comparison shows significant differences which prove the top-layer self-energy to be sensitively dependent on the type of the surface. The more open the surface, the stronger is the narrowing of the toplayer free DOS and thus the narrowing of the self-energy. In Fig. 9 this effect can be seen most clearly at high energies.

It is even more apparent in Fig. 10 where the corresponding imaginary parts are shown in comparison with the imaginary part of the bulk self-energy. The strongest differences with respect to the bulk are found for the (111) surface. As has been mentioned above, the lowered variance of the top-layer free DOS implies a reduced charge density at the surface: $n_{\mathrm{s}}<n<1$. We find $n_{\mathrm{s}}^{(111)}=0.38$ and $n_{\mathrm{s}}^{(110)}=0.52$ to be compared with $n_{\mathrm{s}}^{(100)}=0.56$ and $n=0.6$. According to Eq. (18) this results in a diminished total weight of the imaginary part of the top-layer infinitesimal self-energy. Again the weight is reduced in the occupied part $E<0$ mainly.

In the case of the (100) surface, differences between the local self-energy at the surface and in the bulk are worth mentioning for the top layer only (as discussed above). The same holds for the (110) surface. In the case of the (111) face there are non-negligible differences also for the second layer, while the local self-energy of the third (and all other) surface layer is almost indistinguishable from the bulk self-energy.

Finally let us investigate the effect of the non-local parts of the self-energy on the QDOS. The local QDOS in the first three layers for $U=8$ and at half-filling $(n=1)$ is shown in Fig. 11 for the (110) and in Fig. 12 
for the (111) surface (solid lines). In both cases we find the spectra for all layers to consist of the two high-energy charge-excitation peaks as well as of the resonance at the Fermi level. Compared with the results for the (100) surface (Fig. 8), the fine structure within the resonance is much stronger. This has to be regarded as a pure surface effect since corresponding structures are found in the free DOS at the respective surface as well. The narrowing of the top-layer QDOS is another surface effect that is more pronounced for the (110) and even more for the (111) than for the (100) surface. This is consistent with Eq. (23) when taking into account the coordination numbers of the top-layer sites at the respective surfaces. Due to the imaginary part of the self-energy the differences between the QDOS for different layers are diminished to a large extent compared with $U=0$. If this can be extrapolated to the asymmetric case $n \neq 1$, at least qualitatively, it implies a damping of the surface Friedel oscillations in the layer-dependence of the charge density.

The QDOS shown in Fig. 11 and Fig. 12 has been calculated including the non-local parts of the self-energy up the third neighbor shell (solid lines). This is completely sufficient to obtain fully converged results. For $s=1$, i. e. including the on-site and the nearest-neighbor non-local part only, we get almost the same results. It can thus be concluded that for all surfaces considered the non-local terms beyond nearest neighbors are quite unimportant as concerning their effects on the QDOS. Differences to the fully converged results show up in the case $s=0$, i. e. for the local approximation (dashed lines). However, even for the more open (110) and (111) surfaces the local approximation qualitatively recovers all features in the spectra. Concerning the top-layer QDOS, it is slightly worse than for the (100) surface. For the second- and the third-layer QDOS the errors are comparable to those for the bulk case. Again we find an overestimation of the weight of the resonance around $E=0$ while the weight of the charge-excitation peaks is slightly too small.

\section{CONCLUSIONS}

The behavior of the electronic self-energy and of the quasi-particle density of states at the low-index surfaces of a simple cubic lattice has been investigated within the framework of the semi-infinite Hubbard model. Our main interest has been focussed on the non-locality of the self-energy and its effects on the electronic structure. Within the weak-coupling approach that has been employed for the present study, the non-locality can fully be taken into account. The results for infinitesimally small interaction $U$ are exact. For finite $U$ and at half-filling the SOPT-HF approach is expected to yield qualitatively reliable results at least. SOPT-HF studies have been performed previously for infinitely extended lattices with full translational symmetry (e. g. Refs. [17, 32]).

Let us briefly summarize the results found for the be- havior of the self-energy $\Sigma_{i j}^{(2)}(E)$ at a single-crystal surface: The reduced translational symmetry implies that there are inequivalent sites $i$ and $j$ within the same neighbor shell. This results in a directional dependence of the non-local self-energy parts. The directional dependence has been found to be weak and to be confined to the very surface. The local self-energy $\Sigma_{i i}^{(2)}(E)$ at the surface differs from its bulk value for the top layer only (in the case of the open (111) surface there are non-negligible differences also for the second layer). Differences of the non-local parts $(i \neq j)$ compared with the bulk case are significant if there is at least one site within the top layer. As a consequence of the reduced coordination number of sites at the very surface, there is a narrowing of the free top-layer DOS. This results in a corresponding narrowing of the local top-layer selfenergy. Furthermore, the reduced coordination number results in a diminished (enhanced) surface charge density below (above) half-filling which (in each case) implies a lowered total weight of the imaginary part of the top-layer self-energy. The decrease of the absolute magnitudes of the non-local parts with increasing distance between the sites $i$ and $j$ is as fast as for the bulk, but considerable faster than for the two-dimensional case. All effects mentioned have been found to be the stronger the more open the surface.

The effects of the non-locality of the self-energy on the quasi-particle density of states can be summarized as follows: Qualitatively, all effects that show up in the layer-dependent QDOS at the surface are the same as those for the bulk QDOS: The inclusion of more and more non-local self-energy parts reduces the weight of the quasi-particle resonance at the Fermi level and pronounces the high-energy charge-excitation peaks. Although the top-layer self-energy is sensitively dependent on the type of the surface, the effects of its non-local parts on the QDOS are of the same order of magnitude for the different surfaces considered. In all cases the local approximation is found to yield qualitatively correct results. The errors introduced by the local approximation in the surface region are comparable to those in the bulk. With respect to the QDOS the local approximation is only slightly better than for the two-dimensional case. However, there is one main difference that distinguishes between the $D=2$ lattice on the one hand and the semi-infinite $(D=3)$ lattice on the other: In the latter one gets nearly convergent results by taking into account merely the on-site and the nearest-neighbor self-energy parts. On the contrary, up to $s \approx 5$ shells have to be included to obtain convergence for $D=2$.

It depends on the type of the physical problem to be investigated whether or not one can tolerate the errors introduced by the local approximation. This stresses the need for a quantitative estimate of the effects of nonlocality which has been given here for the semi-infinite Hubbard model. Our study should have shown that if one agrees on the use of the local approximation for the $D=3$ bulk, one may likewise consider a surface without 
the necessity for further justification. In particular, this is important for the application of the dynamical meanfield theory (DMFT) [8, 9] to the semi-infinite Hubbard model. This means to apply those many-body techniques that have been developed for the (approximate or even exact) solution of the infinite-dimensional Hubbard model. For $D=\infty$ the local approximation becomes exact, and there is a universal functional relation between the local self-energy and the QDOS. Within the context of the DMFT the main question thus concerns the effects of the non-local parts of the self-energy on the QDOS which could be estimated within the present study.

\section{ACKNOWLEDGEMENT}

Support of this work by the Deutsche Forschungsgemeinschaft within the Sonderforschungsbereich 290 is gratefully acknowledged.

\section{References}

[1] J. Hubbard, Proc. R. Soc. London, Ser. A 276, 238 (1963).

[2] M. C. Gutzwiller, Phys. Rev. Lett. 10, 159 (1963).

[3] J. Kanamori, Prog. Theor. Phys. (Kyoto) 30, 275 (1963).

[4] E. H. Lieb and F. Y. Wu, Phys. Rev. Lett. 20, 1445 (1968).

[5] J. Sólyom, Adv. Phys. 28, 201 (1979).

[6] F. D. M. Haldane, J. Phys. C 14, 2585 (1981).

[7] W. Metzner and D. Vollhardt, Phys. Rev. Lett. 62, 324 (1989).

[8] D. Vollhardt, In: Correlated Electron Systems, p. 57, Ed. by V. J. Emery. World Scientific Singapore (1993).

[9] A. Georges, G. Kotliar, W. Krauth, and M. J. Rozenberg, Rev. Mod. Phys. 68, 13 (1996).

[10] Y. Nagaoka, Phys. Rev. 147, 392 (1966).

[11] W. Nolting and W. Borgieł, Phys. Rev. B 39, 6962 (1989).

[12] S. Bei der Kellen, W. Nolting, and G. Borstel, Phys. Rev. B 42, 447 (1990).

[13] T. Herrmann and W. Nolting (unpublished).

[14] E. Müller-Hartmann Z. Phys. B 74, 507 (1989).

[15] D. Vollhardt, N. Blümer, K. Held, M. Kollar, J. Schlipf, and M. Ulmke, preprint cond-mat 9701150 (1997).

[16] T. Obermeier, T. Pruschke, and J. Keller (unpublished).

[17] H. Schweitzer and G. Czycholl, Z. Phys. B 83, 93 (1991).

[18] C. J. Powell, J. Electron Spectrosc. Rel. Phen. 47, 197 (1988).

[19] M. Plihal and D. L. Mills, Phys. Rev. B 52, 12813 (1995).

[20] M. Potthoff and W. Nolting, Phys. Rev. B 52, 15341 (1995).

[21] M. Potthoff and W. Nolting, J. Phys.: Condens. Matter 8, 4937 (1996).
[22] M. Potthoff and W. Nolting, Phys. Rev. B 55, 2741 (1997).

[23] M. Potthoff and W. Nolting, Surf. Sci. (in press).

[24] Consider, for example: D. L. Mills, Phys. Rev. B 3, 3887 (1971); T. Kaneyoshi, I. Tamura, and E. F. Sarmento, Phys. Rev. B 28, 6491 (1983); K. Binder and D. P. Landau, Phys. Rev. Lett. 52, 318 (1984); C. Tsallis and E. F. Sarmento, J. Phys. C 18, 2777 (1985); F. AguileraGranja and J. L. Morán-López, Phys. Rev. B 31, 7146 (1985); P. J. Jensen, H. Dreyssé, and K. H. Bennemann, Surf. Sci. 269/270, 627 (1992).

[25] B. Mehlig, H. Eskes, R. Hayn, and M. B. J. Meinders, Phys. Rev. B 52, 2463 (1995).

[26] J. Beenen and D. M. Edwards, Phys. Rev. B 52, 13636 (1995).

[27] K. A. Chao, R. Kishore, and I. C. da Cunha Lima, J. Phys. C 11, L953 (1978).

[28] L. Kleinman, Phys. Rev. 19, 1295 (1979).

[29] G. Treglia, F. Ducastelle, and D. Spanjaard, Phys. Rev. B 21, 3729 (1980).

[30] R. Kishore, Phys. Rev. B 35, 6854 (1987).

[31] R. Taranko and E. Taranko, Physica B 153, 232 (1988).

[32] E. Müller-Hartmann, Z. Phys. B 76, 211 (1989).

[33] G. Bulk and R. J. Jelitto, Phys. Rev. B 41, 413 (1990).

[34] M. M. Steiner, R. C. Albers, and L. J. Sham, Phys. Rev. B 45, 13272 (1992).

[35] G. Schliecker, K.-D. Schotte, B. Horvatić, and V. Zlatić, J. Phys.: Condens. Matter 7, 7969 (1995).

[36] H. Schweitzer and G. Czycholl, Solid State Commun. 74, 735 (1990).

[37] G. Baym and L. P. Kadanoff, Phys. Rev. 124, 287 (1961).

[38] B. Menge and E. Müller-Hartmann, Z. Phys. B 82, 237 (1991).

[39] A. B. Harris and R. V. Lange, Phys. Rev. 157, 295 (1967).

[40] H. Eskes, A. M. Oleś, M. B. J. Meinders, and W. Stephan, Phys. Rev. B 50, 17980 (1994).

[41] H. Kajueter and G. Kotliar, Phys. Rev. Lett. 77, 131 (1996).

[42] M. Potthoff, T. Wegner, and W. Nolting, Phys. Rev. B (in press).

[43] J. M. Luttinger and J. C. Ward, Phys. Rev. 118, 1417 (1960).

[44] J. Mertsching, phys. stat. sol. (b) 82, 289 (1977).

[45] P. W. Anderson, In: Solid State Physics: Advances in Research and Applications, Vol. 14, Ed. by F. Seitz and D. Turnbull, p. 99, Academic New York (1963).

[46] J. Dorantes-Dávila, J. Vega and G. M. Pastor, Phys. Rev. B 47, 12995 (1993); J. Dorantes-Dávila and G. M. Pastor, Phys. Rev. B 51, 16627 (1995); S. Parhofer, R. Pfandzelter and M. Potthoff, Phys. Rev. B 53, 10377 (1996).

[47] A. A. Abrikosow, L. P. Gorkov and I. E. Dzyaloshinski, Methods of Quantum Field Theory in Statistical Physics, Prentice Hall, Englewood Cliffs, NJ (1964).

[48] R. Haydock, In: Solid State Physics, Advances in Research and Applications, Vol. 35, Ed. by H. Ehrenreich and F. Seitz and D. Turnbull. Academic London (1980).

[49] D. G. Pettifor and D. L. Weaire, editors, The Recursion Method and its Applications, Springer Series in SolidState Physics, Vol. 58, Springer Berlin (1984). 
[50] G. D. Mahan, Many-Particle Physics, Plenum New York (1990).

[51] J. M. Luttinger, Phys. Rev. 121, 942 (1961).

[52] J. M. Luttinger, Phys. Rev. 119, 1153 (1960).

[53] This is derived from the real-space representation for $\Sigma_{i i}^{(2)}(E)$ [Eq. (6)] directly. In $\mathbf{k}$ space the fully $\mathbf{k}$ dependent self-energy imaginary part vanishes linearly for $\mathbf{k}$ points on the Fermi "surface", $\operatorname{Im} \Sigma_{\mathbf{k}_{F}}\left(E+i 0^{+}\right) \sim|E|$, and eventually quadratically for all other $\mathbf{k}$ points [17]. For $E \mapsto 0$ the imaginary part of the on-site self-energy $\Sigma_{i i}(E)=\frac{1}{N} \sum_{\mathbf{k}} \Sigma_{\mathbf{k}}(E)$ must therefore vanish "slower" than $\sim E^{2}$ and "faster" than $\sim|E|$, which is consistent with the $\left.\sim E^{2}|\ln | E\right|^{3}$ behavior derived from (6). Thus, contrary to a statement in Ref. [17], the on-site self-energy imaginary part does not vanish quadratically (at $n=1$ ).

[54] The overall three-peak structure of the QDQS has been observed in previous SOPT-HF studies [17, 33. It is remarkable that indications of the splitting into an upper and a lower Hubbard band appear in a weak-coupling approach. This might be ascribed to the fact that (at half-filling) the SOPT-HF self-energy (accidentally) recovers the atomic limit and exhibits the correct highenergy behavior.

[55] SOPT-HF calculations [17] of the bulk self-energy and the bulk spectral density in $\mathbf{k}$ space, $\Sigma_{\mathbf{k}}(E)$ and $\rho_{\mathbf{k}}(E)$, have shown that including only nearest-neighbor corrections to the local approximation leads to $\operatorname{Im} \Sigma_{\mathbf{k}}(E+$ $\left.i 0^{+}\right)>0$ and thereby $\rho_{\mathbf{k}}(E)<0$ in certain (small) regions of the $(\mathbf{k}, E)$ space, i. e. to unphysical results. No such difficulties arise for the $\mathbf{k}$ integrated bulk (and layer-dependent surface) QDOS which is always positive definite for each $s$. 